\title{
Influence des facteurs telluriques abiotiques et de la plante hôte sur l'infection des nématodes phytoparasites du genre Meloidogyne par l'actinomycète parasitoïde Pasteuria penetrans
}

\author{
T Mateille ${ }^{1 *}$, R Duponnois ${ }^{1}$, MT Diop ${ }^{2}$ \\ 1 ORSTOM, laboratoire de nématologie, BP 1386, Dakar; \\ 2 Université Cheikh-Anta-Diop, département de biologie animale, Dakar, Sénégal
}

(Reçu le 12 mai 1995 ; accepté le 13 novembre 1995)

\begin{abstract}
Résumé - Une prospection nématologique a été réalisée dans 4 grandes régions maraîchères du Sénégal (Cap Vert, Pays Sereer, Niayes et Fleuve Sénégal). Pasteuria penetrans, actinomycète parasitoïde des nématodes du genre Meloidogyne, a été rencontré dans toutes les zones prospectées à une fréquence moyenne de $30 \%$ des échantillons, mais il est très peu abondant ( $8 \%$ des juvéniles de Meloidogyne parasités en moyenne). Sa distribution est liée à la texture et à la structure des sols. Les sols sableux sont plus propices que les sols argileux à l'adhésion des spores de l'actinomycète sur les juvéniles du nématode mais, dans les sols sableux, la présence d'argile faciliterait la conservation des spores dans le sol. La plante hôte du nématode joue aussi un rôle sur le développement du parasitoïde soit en améliorant les conditions environnementales de l'adhésion par l'intermédiaire des exsudats racinaires, soit en facilitant sa multiplication dans la cavité générale du nématode infestant.
\end{abstract}

lutte biologique / Meloidogyne spp / Pasteuria penetrans / sol résistant / plante hôte

Summary - Influence of abiotic soil factors and the host plant on the infection of phytoparasitic nematodes of the genus Meloidogyne by the actinomycete parasitoid Pasteuria penetrans. A nematode survey was conducted in the main vegetable-producing areas of Senegal (Cap-Vert, Sereer, Niayes and Senegal valley). Pasteuria penetrans, an actinomycete parasitoid of the nematode genus Meloidogyne, was found in $30 \%$ of the samples all over the areas which were prospected, but it was not abundant (only $8 \%$ of the juveniles of Meloidogyne were infected). Its distribution seems to be influenced by the soil texture and structure. Sandy soils could be more favourable than clay soils for spore attachment of the actinomycete to the nematode juveniles. However, in sandy soils, clay particles, which favour soil aggregation, could retain the spores in the upper soil horizon, with a positive effect of the absorption complex. The host plant of the nematode can influence the development of the parasitoid through the action of the root exudates, which could favour the environmental conditions for attachment, or through the action of plant nutrients, which could increase the multiplication of $\mathrm{P}$ penetrans in the coelomic cavity of the nematode.

biocontrol/Meloidogyne spp / Pasteuria penetrans / suppressive soil/host plant

* Correspondance et tirés à part 


\section{INTRODUCTION}

De nombreux travaux ont été conduits récemment afin d'étudier les potentialités de l'actinomycète Pasteuria penetrans (Sayre et Starr, 1985) comme outil de lutte biologique contre le nématode à galles Meloidogyne spp (Tylenchida). Ainsi, $P$ penetrans a été employé sur diverses cultures annuelles telles que le tabac, la vesce d'hiver et le soja (Brown et al, 1985), l'arachide (Oostendorp et al, 1991), le gombo (Zaki et Maqbool, 1992), la tomate (Channer et Gowen, 1988; Daudi et al, 1990), et des cultures pérennes telles que la vigne (Stirling, 1984) et le kiwi (Verdejo-Lucas, 1992). Cependant, les biologistes observent une grande disparité des résultats due à une efficacité très variable de $P$ penetrans. Aujourd'hui, 2 raisons seulement sont avancées pour expliquer cela : la variabilité interet intra-spécifique de Meloidogyne spp et de $P$ penetrans et, à moindre importance, la sensibilité de $P$ penetrans et de son parasitisme aux conditions climatiques.

L'affinité cuticulaire Meloidogyne-Pasteuria a tout d'abord été étudiée au niveau du genre Meloidogyne (Brown et Smart, 1985) et fut ensuite soupçonnée au niveau de l'espèce (Stirling, 1985). Parallèlement, l'existence d'une variabilité intra-spécifique de $P$ penetrans fut démontrée (Stirling, 1985 ; Bird et al, 1990; Fargette et al, 1994). La reconnaissance cuticulaire serait régie par des mécanismes biochimiques impliquant les protéines de surface des spores de $P$ penetrans et les composés glucidiques cuticulaires des nématodes (Persidis et al, 1991 ; Davies et al, 1992 ; Davies et Danks, 1993).

Les 2 facteurs climatiques les plus étudiés sont l'humidité et la température. Bien que les spores de $P$ penetrans puissent survivre longuement dans des sols secs (Stirling et Wachtel, 1980), l'humidité des sols (Brown et Smart, 1984) et plus encore l'alternance de dessiccation et d'humidification des spores (Oostendorp et al, 1990) sont favorables au processus d'adhésion. Dans des conditions climatiques tempérées, un accroissement de la température jusqu'à $30^{\circ} \mathrm{C}$ favorise l'adhésion des spores aux nématodes (Stirling, 1981; Hatz et Dickson, 1992), phénomène expliqué par une augmentation de la mobilité des juvéniles de Meloidogyne, donc par une probabilité de rencontre plus élevée entre les juvéniles et les spores de $P$ penetrans (Stirling et al, 1990). Cependant, l'adhésion des spores est toujours possible après un traitement à des températures létales supérieures à $100^{\circ} \mathrm{C}$ (Dutky et
Sayre, 1978 ; Stirling et al, 1986) : l'adhésion ne procède donc pas seulement d'un mécanisme de reconnaissance biochimique thermosensible.

Mais la variabilité spécifique du nématode et du parasitoïde et les conditions climatiques étudiées ne suffisent pas pour expliquer la fluctuation de l'efficacité du parasitoïde. L'objectif de cette étude, basée sur une enquête nématologique, microbiologique, botanique et pédologique, est de montrer que le développement de Pasteuria penetrans dépend d'autres éléments de son habitat tellurique comme l'activité racinaire de la plante hôte du nématode et la composition physico-chimique des sols.

\section{MATÉRIEL ET MÉTHODES}

\section{Enquêtes sur la répartition du nématode Meloidogyne spp et de son parasitoïde Pasteuria penetrans}

Des prospections ont été effectuées dans 4 grandes zones de cultures maraîchères du Sénégal, entre les mois de mars et juillet 1993. Ces zones sont la presqu'île du Cap Vert, la région des Niayes, le pays Sereer et la vallée occidentale du fleuve Sénégal (fig 1, tableau I).

Dans chaque parcelle visitée, des prélèvements de sol et de racines ont été effectués le long d'un transect à une densité de 3 prélèvements sur $10 \mathrm{~m}$ rassemblés en un seul échantillon de référence. En tout, 210 échantillons ont été prélevés. La répartition de l'échantillonnage reflétait la densité régionale des surfaces cultivées en cultures maraîchères : $37 \%$ dans la région du Cap Vert, $24 \%$ dans la région des Niayes, $29 \%$ dans le pays Sereer et $10 \%$ dans la vallée du Sénégal.

Les cultures les plus importantes ont été échantillonnées à la fréquence suivante : tomate (Lycopersicon esculentum) : 32\% ; gombo (Hibiscus esculentus) : $10 \%$; aubergine africaine (Solanum aethiopicum) : $9 \%$; pomme de terre (Solanum tuberosum) : $8 \%$; chou (Brassica oleracea) : $7 \%$; pastèque (Citrulus vulgaris) : $6 \%$.

Les nématodes ont été extraits du sol et des racines selon les méthodes de Seinhorst $(1950,1962)$. Le dénombrement des juvéniles de Meloidogyne spp a été ramené au $\mathrm{dm}^{3}$ de sol (MeS) ou au gramme de racines sèches (MeR)

Les populations de Pasteuria penetrans ont été évaluées après comptage :

- des spores de $P$ penetrans (organismes en forme de coupelles de $4 \mu \mathrm{m}$ de diamètre environ facilement reconnaissables) sur les juvéniles de Meloidogyne (grossissement : $\times 120$ ). Le nombre de spores a été ramené à 3 classes d'abondance : aucune spore ; 1 à 


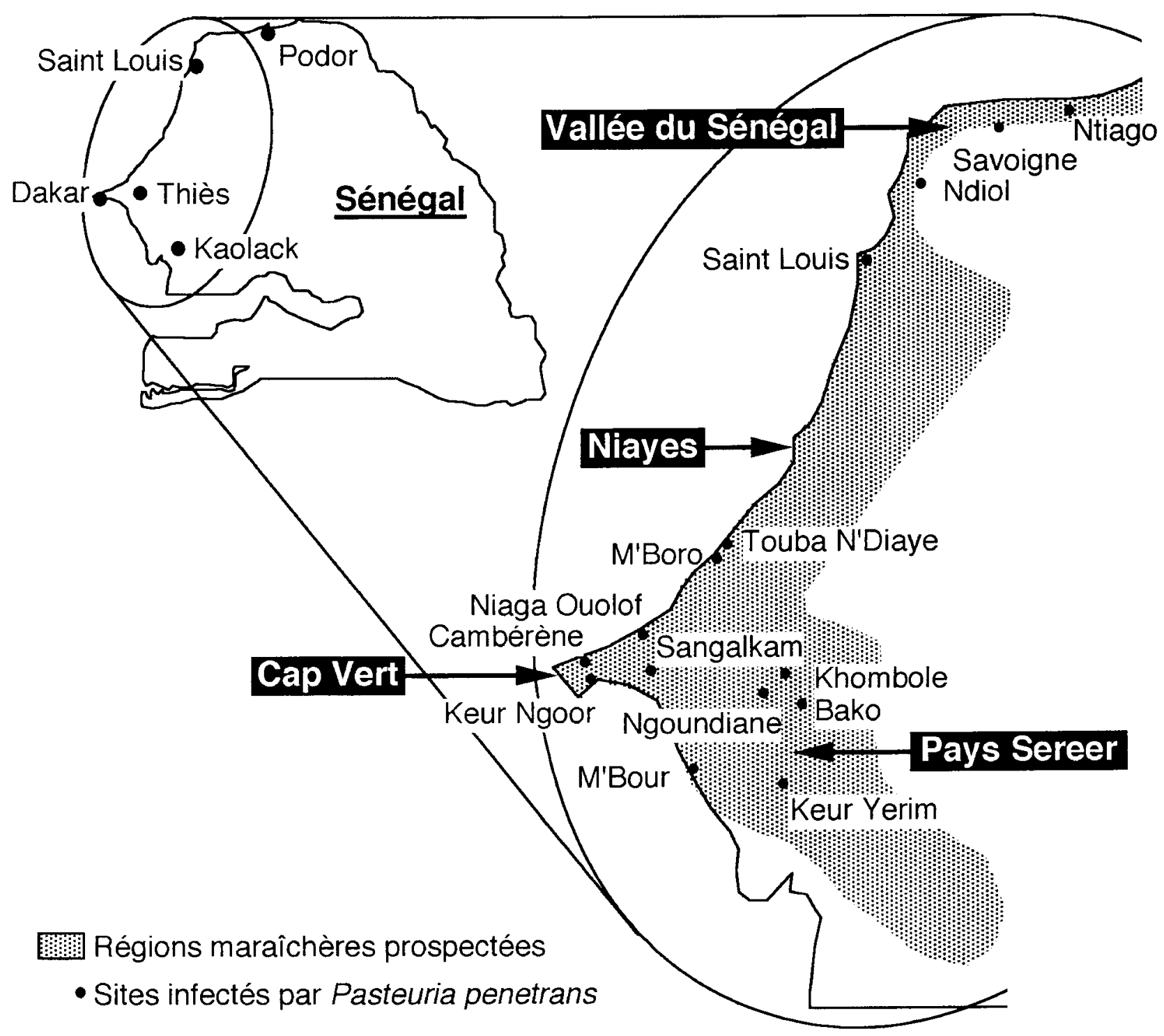

Fig 1. Répartition géographique de Pasteuria penetrans dans les zones maraîchères prospectées au Sénégal.

Tableau I. Caractéristiques agro-pédo-climatiques des zones maraîchères prospectées.

\begin{tabular}{|c|c|c|c|c|}
\hline Régions & Types de sol & Texture & Températures a & Pluviométries a \\
\hline Cap-Vert & Ferrugineux & Sablo-limoneuse & $<25^{\circ} \mathrm{C}$ & $300-400 \mathrm{~mm}$ \\
\hline Niayes & Minéraux bruts & Sableuse & $<25^{\circ} \mathrm{C}$ & $250-400 \mathrm{~mm}$ \\
\hline Pays Sereer & $\begin{array}{c}\text { Ferrugineux } \\
\text { vertisols }\end{array}$ & $\begin{array}{l}\text { Sablo-limoneuse } \\
\text { Sablo-limoneuse }\end{array}$ & $26-27^{\circ} \mathrm{C}$ & $400-600 \mathrm{~mm}$ \\
\hline Vallée Sénégal & $\begin{array}{c}\text { Hydromorphes } \\
\text { salins }\end{array}$ & $\begin{array}{l}\text { Argileuse } \\
\text { Argileuse }\end{array}$ & $25-27^{\circ} \mathrm{C}$ & $<200 \mathrm{~mm}$ \\
\hline
\end{tabular}


10 spores par juvénile ; plus de 10 spores par juvénile.

- des juvéniles de Meloidogyne infectés (grossissement : $x$ 50). Le pourcentage de juvéniles infectés a été ramené à 4 classes d'abondance : $0 \% ; 0<a<$ $5 \% ; 5 \leq b<10 \% ; c \geq 10 \%$.

\section{Analyses de sol}

Les analyses ont été effectuées sur tous les échantillons de sol provenant des parcelles qui contenaient $P$ penetrans et sur tous les échantillons des parcelles les plus proches qui n'en contenaient pas (échantilions appariés).

Des analyses granulométriques ont permis de déterminer les proportions d'argile $(\operatorname{Arg}=0-2 \mu \mathrm{m})$, de limon fin ( $\mathrm{LiF}=2-20 \mu \mathrm{m})$ et grossier ( $\mathrm{LiG}=$ 20-50 $\mu \mathrm{m})$, de sable fin (SaF $=50-200 \mu \mathrm{m})$ et grossier $(\mathrm{SaG}=200-2000 \mu \mathrm{m})$.

Des analyses physico-chimiques ont permis de déterminer le taux de matière organique (MOr), le point de flétrissement ( $\mathrm{pF} 4,2$ ), le $\mathrm{pH} \mathrm{H}_{2} \mathrm{O}(\mathrm{pH})$, les concentrations en phosphore assimilable (Pas), en bases ( $\mathrm{Ca}, \mathrm{Mg}, \mathrm{Na}, \mathrm{K}$ ) échangeables $(\mathrm{E})$ et totales $(\mathrm{T})$, la capacité d'échange (CEC), la conductivité (Con) et l'humidité (Hum).

\section{Analyses des données}

\section{Enquêtes}

La distribution statistique des populations de Meloidogyne spp infectées ou pas par $P$ penetrans a été établie à partir des diagrammes de fréquence (pourcentage des échantillons dans lesquels le nématode est présent) et d'abondance (moyenne des effectifs des nématodes dans les échantillons où ils sont présents) définies par Fortuner et Merny (1973).

\section{Distribution de $\boldsymbol{P}$ penetrans}

Les résultats des analyses de sol ont été regroupés dans un tableau comportant en colonne les différentes variables mesurées et en ligne les individus (échantillons analysés). Un autre tableau de données regroupait en colonne la présence $(+)$ ou l'absence $(-)$ de juvéniles de Meloidogyne spp infectés par $P$ penetrans. L'analyse des correspondances a été réalisée grâce à la méthode d'analyse en composantes principales (ACP normée). Puis une autre analyse a été effectuée seulement sur les échantillons infestés par $P$ penetrans. Cette fois, le tableau regroupait en colonne les proportions des juvéniles infectés établies en 3 classes $(0<a<5 \% ; 5 \leq b<10 \% ; c \geq 10 \%)$. Ces analyses ont été effectuées et représentées graphiquement selon la méthode de Thioulouse (1989).

\section{RÉSULTATS}

\section{Distribution de Meloidogyne spp et de Pasteuria penetrans au Sénégal}

Les populations de Meloidogyne spp non infectés par $P$ penetrans sont très fréquentes et abondantes dans toutes les régions prospectées, mais on les trouve plus fréquemment dans les racines que dans le sol (fig 2). Si les niveaux des populations telluriques sont comparables d'une région à l'autre, en revanche les fréquences sont différentes: les populations de Meloidogyne sont plus répandues dans la région du Cap Vert que dans la vallée du Sénégal, dans le pays Sereer et dans les Niayes. Au plan racinaire, la fréquence des populations est plus faible dans la vallée du Sénégal que dans les 3 autres régions. En revanche, à fréquence égale, l'abondance des populations est plus élevée dans la région du Cap Vert que dans les Niayes et le pays Sereer.

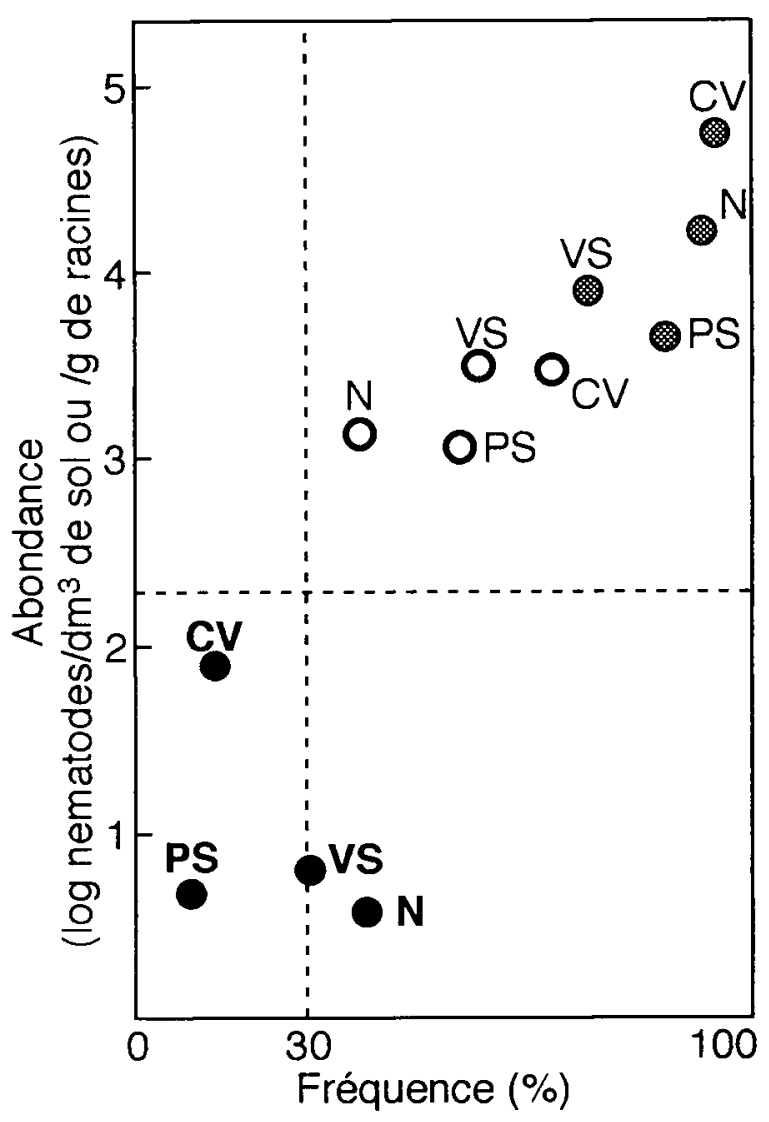

Fig 2. Distribution des juvéniles de Meloidogyne spp infectés ou non par Pasteuria penetrans ( $\mathrm{CV}=\mathrm{Cap}$ Vert $; \mathrm{N}=$ Niayes; $\mathrm{PS}=$ pays Sereer, VS = vallée du Sénégal ; points grisés = populations racinaires de juvéniles non infectés ; points blancs $=$ populations telluriques de juvéniles non infectés ; points noirs = populations telluriques de juvéniles infectés). 
Pasteuria penetrans est présent dans toutes les régions agroclimatiques de culture maraîchère prospectées au Sénégal (fig 1). Les populations de Meloidogyne spp infectés par $P$ penetrans sont beaucoup moins fréquentes et abondantes que les populations de juvéniles non infectés. Cependant, la fréquence moyenne des populations infectées sur l'ensemble des régions prospectées est de $30 \%$. À fréquence équivalente, les juvéniles de Meloidogyne infectés par $P$ penetrans sont beaucoup plus abondants dans la presqu'île du Cap Vert que dans le pays Sereer. A abondance égale, ils sont plus répandus dans les Niayes (fréquence identique à celle des juvéniles de Meloidogyne non infectés) que dans la vallée du Sénégal et le pays Sereer.

\section{Influence des facteurs telluriques abiotiques sur la distribution de Pasteuria penetrans}

La première analyse en composante principale effectuée pour caractériser les échantillons seion l'absence $(-)$ ou la présence $(+)$ de $P$ penetrans montre que les facteurs 1 et 2 du plan factoriel expliquent $57,5 \%$ de la variabilité des données. Les infestations en Meloidogyne spp dans le sol (MeS) et dans les racines (MeR) ne sont corrélées à aucun des 2 facteurs. La majorité des variables est située dans les valeurs négatives du facteur 1 (fig 3A). Les variables les plus corrélées sont aussi bien des variables chimiques: capacité d'échange (CEC), toutes les bases échangeables ( $\mathrm{MgE}, \mathrm{CaE}, \mathrm{NaE}, \mathrm{K}-\mathrm{E}), 3$ bases totales (MgT, NaT et $\mathrm{K}-\mathrm{T}$ ) et la conductivité (Con), que des variables structurales et texturales : point de flétrissement ( $\mathrm{pF} 4)$, proportion d'argile (Arg) et de limons fins (LiF). Sur le facteur 2, la plupart des variables chimiques sont situées dans les valeurs négatives, alors que la plupart des variables texturales sont situées dans les valeurs positives. Les proportions en sables fins (SaF) et grossiers (SaG) s'opposent sur ce facteur.

Les points correspondant aux échantillons dans lesquels $P$ penetrans est absent $(-)$ et à ceux dans lesquels $P$ penetrans est présent $(+)$ semblent être répartis de manière identique dans tout le plan factoriel F1 x F2 (fig 3B). Mais la distribution gaussienne des points sur le facteur 1 (fig $3 C$ ) indique que les points $(-)$ sont répartis tout le long du facteur 1 , alors que les points $(+)$ sont plus concentrés. Les pics de distribution des points $(-)$ et $(+)$ sont légèrement décalés, les premiers vers les valeurs négatives de $\mathrm{F} 1$, les seconds vers les valeurs positives.
La seconde analyse effectuée pour caractériser les échantillons selon l'abondance de $P$ penetrans dans les échantillons où l'actinomycète est présent ne montre pas de variation importante de la situation des variables dans le plan factoriel par rapport à la première analyse (fig 4A). En revanche, elle indique que la majorité des points correspondant à la classe d'abondance la plus élevée (c) est corrélée aux valeurs positives du facteur 1 , alors que les points correspondant aux classes inférieures ( $a$ et $b$ ) se distribuent à la fois dans les valeurs positives et négatives de ce facteur (fig 4B). Le facteur 2 sépare les points correspondant à la classe d'abondance la plus faible (a), situés en majorité dans les valeurs négatives du facteur, des points correspondant à la classe d'abondance intermédiaire (b) situés en majorité dans les valeurs positives du facteur. Les points de la classe d'abondance la plus élevée (c) sont répartis sur tout le facteur 2 .

Le retour aux données de base (fig 5) montre que, par rapport au facteur 1 , la classe d'abondance en $P$ penetrans la plus élevée (c) correspond significativement $(p<0,05)$ à des valeurs faibles de la capacité d'échange, des concentrations en magnésium, sodium, calcium et potassium échangeables, de la conductivité, du point du flétrissement $\mathrm{pF} 4,2$ et de la proportion en argiles, limons fins et sables grossiers, alors que les classes d'abondance les plus basses ( $a$ et $b$ ) correspondent à des valeurs élevées de ces variables. Les autres variables varient dans le même sens mais pas de manière significative. Le facteur 2 ne sépare que les classes d'abondance les plus faibles (a et b) : la classe (a) correspond significativement à des proportions élevées en sables grossiers et en matière organique mais faibles en sables fins, et à des concentrations élevées en phosphore assimilable et en calcium total, et inversement pour la classe d'abondance (b).

Les diagrammes représentant les caractéristiques physico-chimiques des sols en fonction de la proportion de sables grossiers dans les échantillons où $P$ penetrans est présent indiquent que 3 grands types de sols ont été échantillonnés (fig 6) : i) des sols à texture fine avec moins de $30 \%$ de sables grossiers et une forte proportion d'argiles, de limons fins et grossiers, de sables fins, de matière organique, une capacité d'échange et des proportions de bases échangeables élevées ; ii) des sols à texture moyenne avec 30 à $45 \%$ de sables grossiers et avec une très faible proportion d'argiles et de matière organique et une capacité d'échange très faible ; iii) des sols à texture grossière avec plus de $45 \%$ de sables grossiers, mais dans lesquels les proportions d'argiles et de 


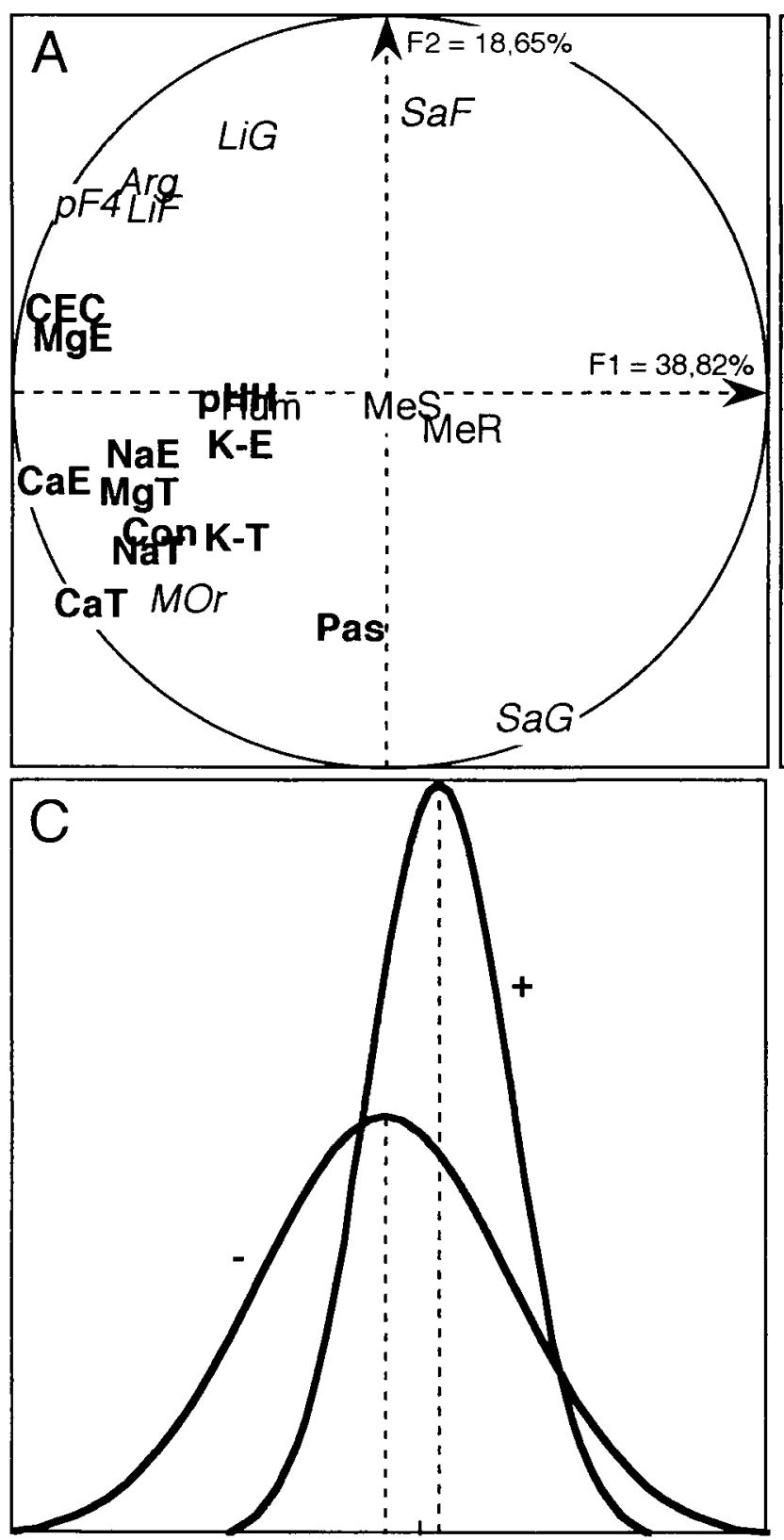

matière organique croissent ainsi que les composantes chimiques. Quand on rapporte la proportion des 3 classes d'abondance en juvéniles de Meloidogyne spp parasitées par $P$ penetrans sur ces 3 types de sols, on constate que la classe d'abondance la plus faible (a) est aussi fréquente quelle que soit la texture. En revanche, la classe d'abondance la plus élevée (c) est absente des sols à texture fine très argileux alors qu'elle apparaît et augmente dans les 2 autres types de sols à texture sableuse grossière.

\section{Influence de la plante hôte de Meloidogyne spp sur la distribution de Pasteuria penetrans}

La seconde analyse en composante principale effectuée pour caractériser les échantillons selon

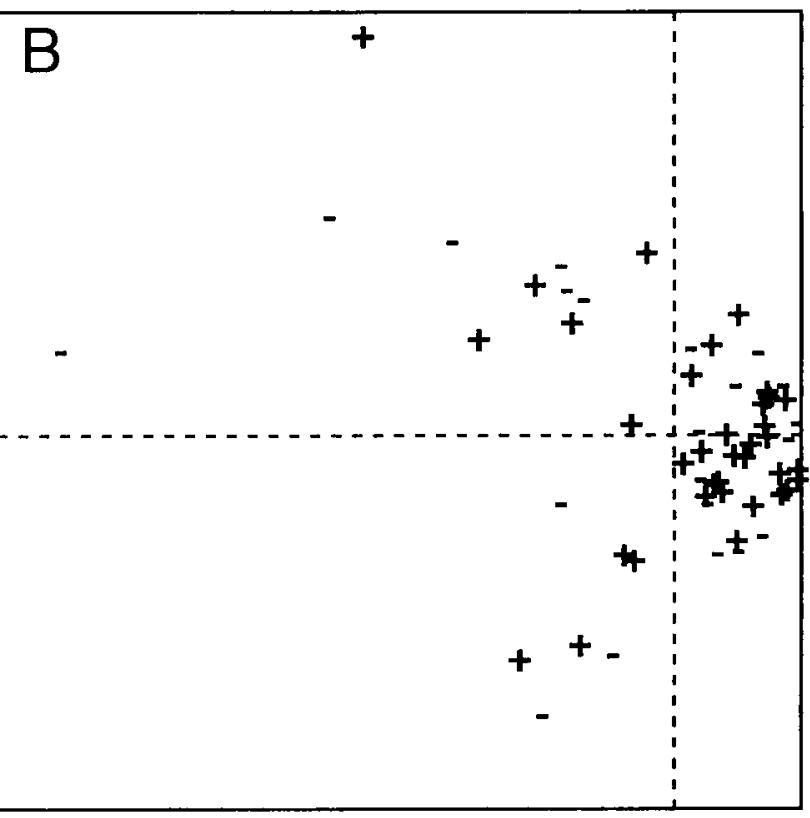

Fig 3. Caractéristiques des échantillons de sol avec ou sans Pasteuria penetrans. A = cercle de corrélation des variables de I'ACP ( $\mathrm{Arg}=$ argiles $; \mathrm{LiF}=$ limons fins $; \mathrm{LiG}=$ limons grossiers $; \mathrm{SaF}=$ sables fins ; $\mathrm{SaG}=$ sables grossiers $; \mathrm{MOr}=$ matière organique ; $\mathrm{pF} 4=\mathrm{pF} 4,2 ; \mathrm{pHH}=\mathrm{pH} \mathrm{H} 2 \mathrm{O} ;$ Pas = phosphore assimilable ; $\mathrm{CaE}, \mathrm{MgE}, \mathrm{NaE}, \mathrm{K}-\mathrm{E}$ = bases échangeables ; $\mathrm{CaT}, \mathrm{MgT}, \mathrm{NaT}, \mathrm{K}-\mathrm{T}=$ bases totales $; \mathrm{CEC}=$ capacité d'échange $;$ Con = conductivité $;$ Hum = humidité $\mathrm{MeR}=$ effectif de juvéniles de Meloidogyne dans les racines $;$ MeS = effectif de juvéniles de Meloidogyne dans le sol). $\mathbf{B}=$ dispersion des points dans le plan factoriel F1 x F2 en fonction de l'absence (-) et de la présence $(+)$ de Pasteuria penetrans. $\mathbf{C}=$ dispersion gaussienne des points sur le facteur $\mathrm{F} 1$ en fonction de l'absence $(-)$ et de la présence $(+)$ de Pasteuria penetrans.

l'abondance de $P$ penetrans dans les échantillons où l'actinomycète est présent (fig 4) montre que :

- la plupart des points correspondant à des échantillons prélevés sur aubergine africaine ( $S$ aethiopicum) sont situés dans les valeurs positives du facteur 1 et correspondent en majorité à la classe de $P$ penetrans la plus élevée (c).

- la plupart des points correspondant à des échantillons prélevés sur tomate sont situés tout le long du facteur 1 et correspondent en majorité aux classes d'abondance de $P$ penetrans les plus faibles ( $a$ et $b$ ).

Ceci est confirmé en comparant la proportion des juvéniles infectés par $P$ penetrans sous les cultures les plus fréquemment échantillonnées (fig 7). Cette proportion est significativement plus 


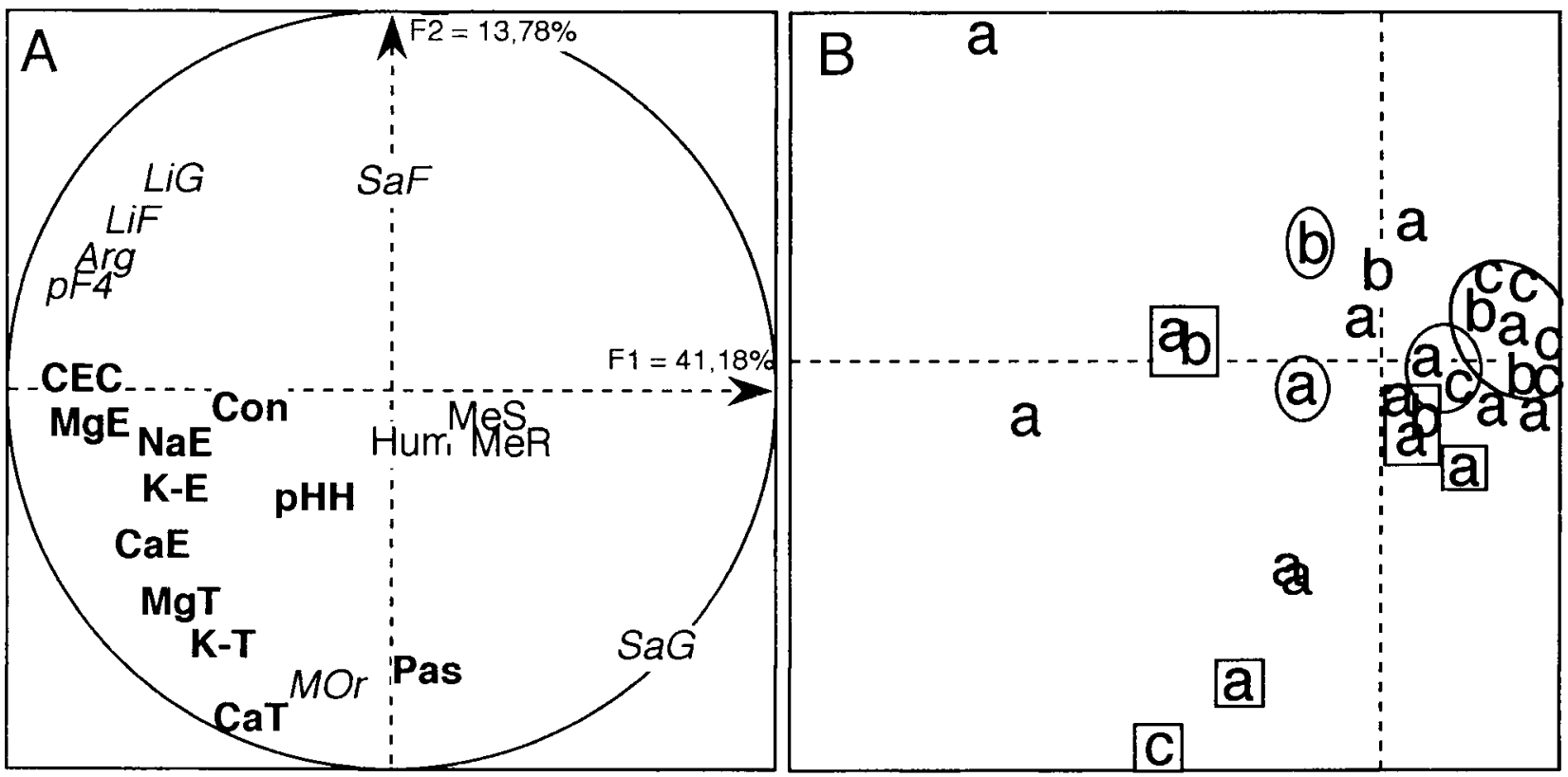

Fig 4. Caractéristiques des échantillons de sol contenant Pasteuria penetrans. $\mathbf{A}=$ cercle de corrélation des variables de I'ACP (légende des variables in fig 3). $\mathbf{B}=$ dispersion des points dans le plan factoriel F1 x F2 en fonction de la proportion de juvéniles de Meloidogyne spp infectés par Pasteuria penetrans dans les échantillons où il est présent $(0<a<5 \% ; 5 \leq b<10 \% ; c \geq 10 \% ;$ plantes hôtes correspondantes : les points entourés d'un rectangle correspondent à des échantillons prélevés sur tomate ; les points entourés d'une ellipse correspondent à des échantillons prélevés sur aubergine africaine Solanum aethiopicum).

élevée sous culture d'aubergine africaine $(S$ aethiopicum) que sous culture de tomate. Elle est encore beaucoup plus faible sous culture de pomme de terre et de chou. En revanche, $P$ penetrans n'a été trouvé qu'une seule fois sous culture de gombo et sous culture de pastèque (pourtant rencontrées presque aussi souvent que l'aubergine africaine) avec respectivement 2,6 et $7,9 \%$ de juvéniles infectés.

\section{DISCUSSION}

L'étude de la distribution des populations de Meloidogyne spp dans les 4 régions de culture échantillonnées montre que les juvéniles sont beaucoup plus souvent présents dans les racines que dans le sol. Ceci a déjà été observé en comparant l'influence de 2 types de sols sur l'équilibre entre les populations telluriques et racinaires (Mateille, résultats non publiés) : pour une même infestation racinaire, la population tellurique correspondante est plus importante dans des sols sablo-limoneux (comme dans le pays Sereer) et dans des sols sableux (comme dans la région des Niayes). Par conséquent, l'adhésion des spores de $P$ penetrans se réalisant dans le sol, la proportion de juvéniles de Meloidogyne infectés est faible dans les sols très sableux.
Cependant, cette étude montre que l'incidence du sol ne se situe pas seulement au niveau du type de sol mais, plus précisément, au niveau de leurs caractéristiques physico-chimiques, c'est-àdire non pas à une échelle régionale mais plutôt à une échelle locale, puisque l'étude de la présence/absence de $P$ penetrans a été réalisée sur des échantillons appariés provenant de parcelles voisines, donc de types de sol identiques. Le fait que $P$ penetrans puisse être absent quelle que soit la composition physico-chimique des sols (les points (-) sont répartis tout le long de l'axe F1) est une évidence. En revanche, la présence ainsi que l'abondance croissante du parasitoïde sont liées à une composition physico-chimique particulière. Les 2 premières analyses en composantes principales illustrent l'effet global des caractéristiques des sols. Elles nous indiquent l'importance majeure de l'ensemble des composantes du complexe absorbant avec, en premier lieu, les argiles et, ensuite, les éléments qui leurs sont liés, à savoir le point de flétrissement et les bases échangeables. Cet effet global se traduit par le fait que $P$ penetrans serait d'autant moins abondant que les sols seraient argileux. En fait, cet effet masque 2 effets particuliers qui sont révélés par la correspondance entre les classes d'abondance en $P$ penetrans et les textures des sols. II apparaît effectivement que les sols très 


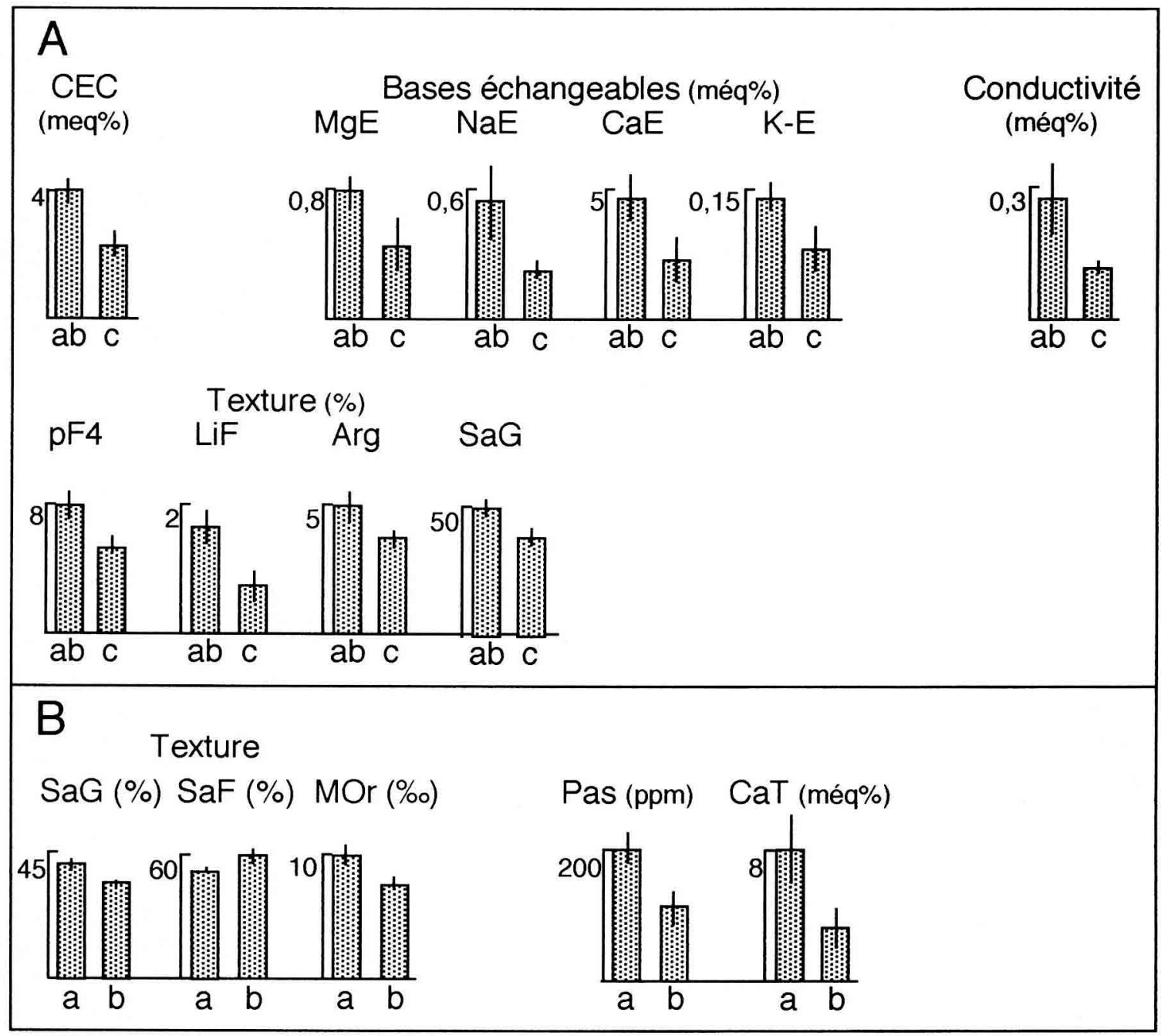

Fig 5. Correspondances significatives $(p \leq 0,05)$ entre les proportions de juvéniles de Meloidogyne spp infectés par Pasteuria penetrans $(0<\mathrm{a}<5 \% ; 5 \leq \mathrm{b}<10 \% ; \mathrm{c} \geq 10 \%)$ et les facteurs telluriques abiotiques par rapport au facteur 1 (A) et au facteur 2 (B) de I'ACP (les barres représentent les intervalles de confiance).

argileux sont peu propices à $P$ penetrans alors que les sols sableux le sont. Mais les sols sableux sont d'autant plus favorables à $P$ penetrans qu'ils renferment plus d'argiles et de matière organique. On peut alors distinguer 2 effets : structural et électrostatique. En effet, quand elles représentent la fraction fine majoritaire, les argiles rendent les sols très compacts et limitent ainsi la migration des juvéniles de Meloidogyne et, par conséquent, réduisent la probabilité de rencontre des juvéniles avec les spores libres de $P$ penetrans. D'autre part, elles peuvent «emprisonner" les spores dans le rhizoplan au contact de la galle racinaire dont elles sont issues, les rendant indisponibles à l'adhésion avec des juvéniles migrant à des distances plus éloignées des racines. Au contraire, les sols de texture plus grossière facilitent le déplacement des juvéniles de Meloidogyne (Prot, 1979), augmentant ainsi les chances de rencontre entre les 2 organismes.
Mais, dans ces sols, les argiles, qui augmentent la porosité, ont un effet bénéfique sur la conservation des spores. En effet, les spores libres de $P$ penetrans sont assimilables à des particules de $4 \mu \mathrm{m}$ de diamètre environ (Sayre et Wergin, 1977) qui vont, avec les mouvements de l'eau (l'irrigation moyenne des cultures maraîchères correspond à environ $10 \mathrm{~mm}$ par jour), être plus ou moins "lessivées" selon l'importance du complexe absorbant. Les sols grossiers à structure particulaire (absence d'argiles) favoriseraient la fuite passive des spores hors de l'horizon rhizosphérique (Oostendorp et al, 1990) où se situent les nématodes alors que les mêmes sols à structure agrégée les retiendraient. De même, bien que la charge électrique globale externe des spores de $P$ penetrans soit inconnue, les colloïdes pourraient entraîner, selon l'état de saturation du complexe absorbant en cations bivalents $\mathrm{Ca}^{2+}$ et $\mathrm{Mg}^{2+}$, une rétention plus ou moins forte 


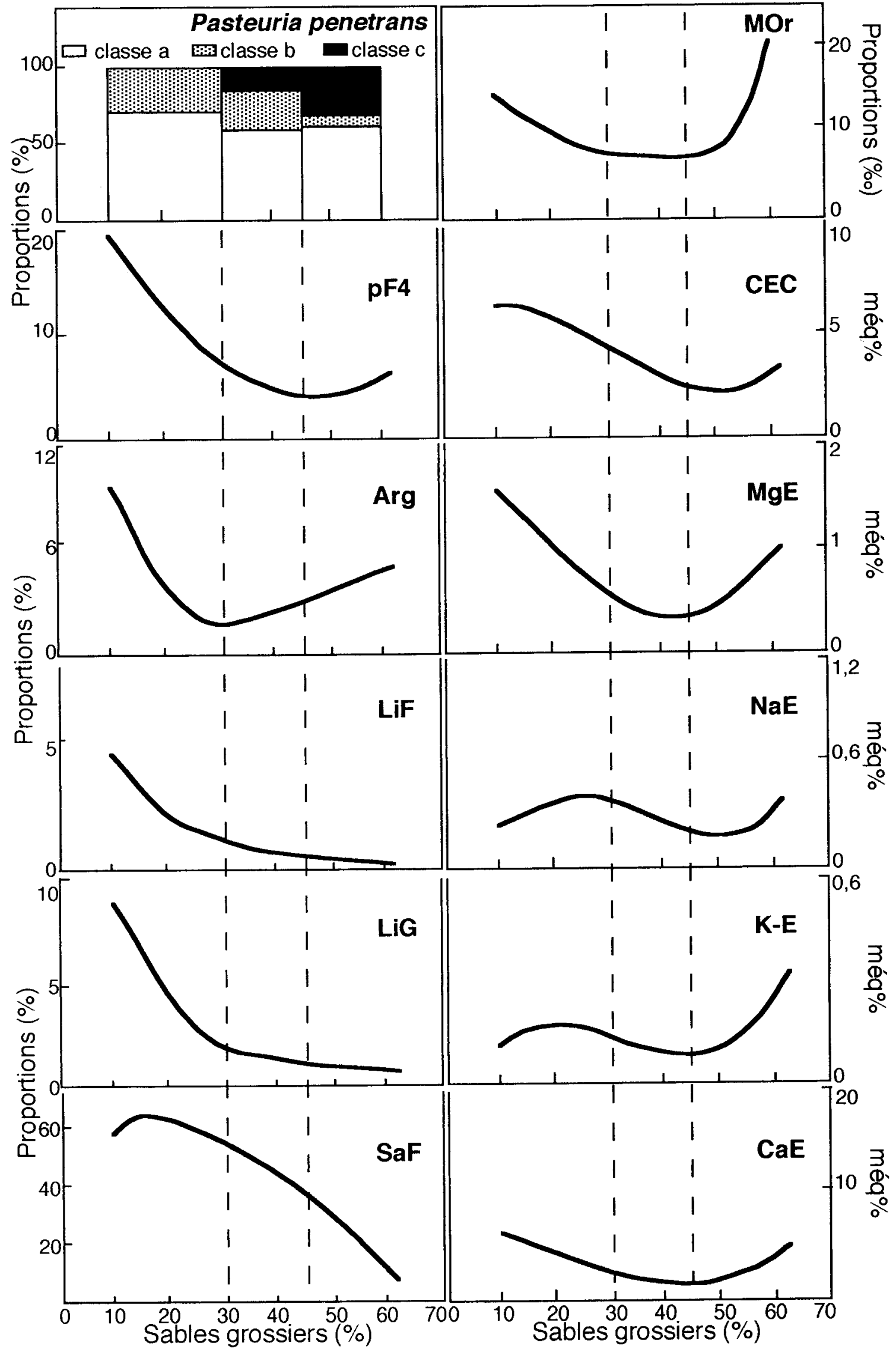

Fig 6. Correspondance entre la fréquence des classes d'abondance en Pasteuria penetrans et les caractéristiques physico-chimiques des échantillons de sols (légende des variables dans la figure 3 ). 


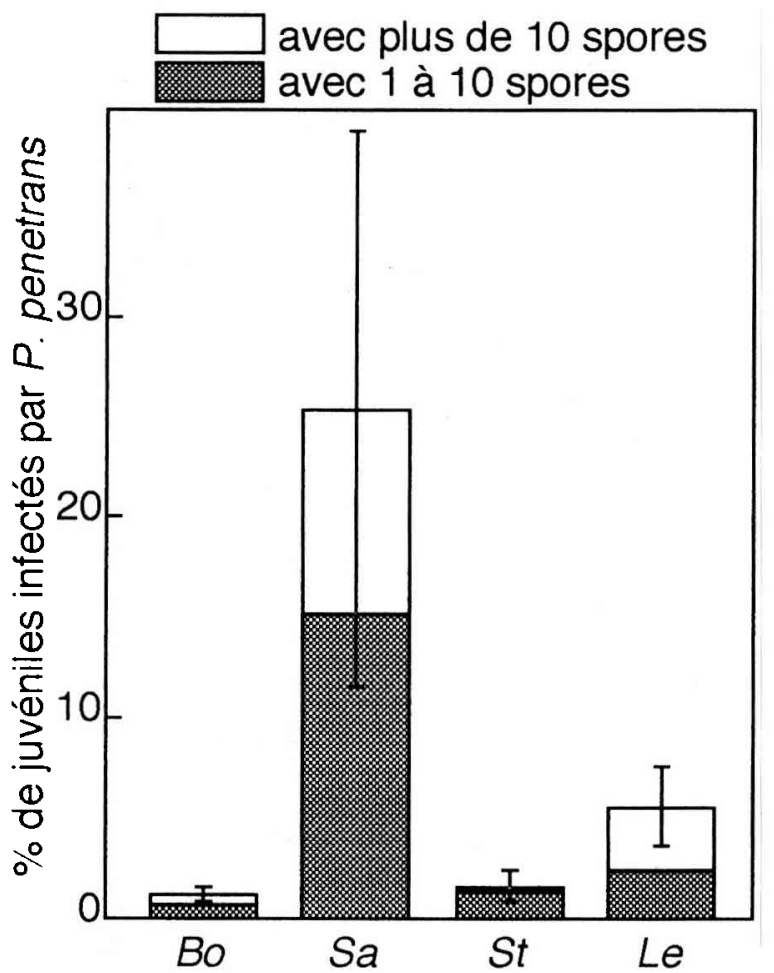

Fig 7. Fréquences des juvéniles de Meloidogyne spp infectés par Pasteuria penetrans sous culture de chou Brassica oleracea $(\mathrm{Bo})$, d'aubergine africaine Solanum aethiopicum (Sa), de pomme de terre Solanum tuberosum (St) et de tomate Lycopersicon esculentum (Le) (les barres représentent les intervalles de confiance de la moyenne des pourcentages totaux de juvéniles infectés).

des spores sur les feuillets argileux. La disponibilité maximale de $P$ penetrans dépendrait donc d'un équilibre texture/structure optimal du sol.

Parmi les cultures échantillonnées, les plantes telles que l'aubergine africaine, la tomate, la pomme de terre, le gombo ou la pastèque sont très sensibles à Meloidogyne spp. Or l'abondance en $P$ penetrans diffère selon l'hôte du nématode. Cet effet peut s'expliquer soit par l'action des exsudats racinaires, soit par la qualité nutritionnelle des plantes. En effet, les exsudats sont attractifs pour les nématodes et la pénétration des nématodes dans une racine induit une augmentation de la concentration de certains éléments, tels que des polysaccharides (Wang et Bergeson, 1974), dans les exsudats. Dans le sol, ces composés pourraient faciliter l'adhésion des spores de $P$ penetrans sur la cuticule des juvéniles de Meloidogyne par l'intermédiaire de ponts saccharidiques. Par ailleurs, compte tenu que la germination de $P$ penetrans dans le nématode ne se réalise qu'une fois le nématode fixé sur son site nourricier racinaire (Sayre, 1980), la qualité nutritionnelle de la plante pour le nématode pourrait avoir une incidence sur le développement et la multiplication intracœlomique de $P$ penetrans.

\section{CONCLUSION}

Outre les problèmes d'affinité biochimique entre le nématode Meloidogyne spp et l'actinomycète parasitoïde $P$ penetrans et la sensibilité du parasitisme aux conditions climatiques, cette enquête montre que l'hétérogénéité de la répartition et de l'efficacité de $P$ penetrans est aussi liée aux caractéristiques des sols et à la plante hôte du nématode. Les sols sableux contenant une faible proportion d'argiles sont les mieux adaptés à son développement. Les exsudats racinaires pourraient participer à l'efficience du parasitoïde. En conséquence, l'amélioration, ou le maintien, des qualités suppressives des sols à $P$ penetrans nécessitent une meilleure connaissance des interactions mésologiques et biologiques qui régissent les mécanismes d'antibiose entre $P$ penetrans et Meloidogyne spp.

\section{REMERCIEMENTS}

L'enquête faunistique a bénéficié d'un financement de la Communauté européenne (EC Project STD $3 n^{\circ}$ TS3 * CT92-0098 : «Biocontrol of damaging root-knot nematode (Meloidogyne spp) pests of staple food and cash crops by including suppressive soils with the bacterial parasite Pasteuria penetrans"). Les auteurs remercient $P$ Cadet (laboratoire de nématologie) et $\mathrm{JL}$ Duprey (laboratoire de chimie), ORSTOM, Dakar, pour leur assistance technique.

\section{RÉFÉRENCES}

Bird AF, Brisbane PG, McClure SG, Kimber RWL (1990) Studies on the properties of the spores of some populations of Pasteuria penetrans. J Inverteb Pathol 55, 169-178

Brown SM, Smart GC (1984) Attachment of Bacillus penetrans to Meloidogyne incognita. Nematropica 14, 171-172

Brown SM, Smart GC (1985) Root penetration by Meloidogyne incognita juveniles infected with Bacillus penetrans. J Nematol 17, 123-126

Brown SM, Kepner JL, Smart GC (1985) Increased crop yields following application of Bacillus penetrans to field plots infested with Meloidogyne incognita. Soil Biol Biochem 17, 483-486

Channer AG, Gowen SR (1988) Preliminary studies on the potential of Pasteuria penetrans to control Meloidogyne species. Proc Brighton Crop Protect Conf, Pests Dis 3, 1209-1214

Daudi AT, Channer AG, Ahmed R, Gowen SR (1990) Pasteuria penetrans as a biocontrol agent of Meloidogyne javanica in the field in Malawi and in 
microplots in Pakistan. Proc Brighton Crop Protect Conf Pests Dis 1, 253-257

Davies KG, Danks C (1993) Interspecific differences in the nematode surface coat between Meloidogyne incognita and $M$ arenaria related to the adhesion of the bacterium Pasteuria penetrans. Parasitology $105,475-480$

Davies KG, Robinson MP, Laird V (1992) Proteins involved in the attachment of a hyperparasite Pasteuria penetrans to its plant-parasitic nematode host, Meloidogyne incognita. J Inverteb Pathol 59, 18-23

Dutky EM, Sayre RM (1978) Some factors affecting infection of nematodes by bacterial spore parasite Bacillus penetrans. J Nematol 10, 285

Fargette M, Davies KG, Robinson MP, Trudgill DL (1994) Characterization of resistance-breaking Meloidogyne incognita-like populations using lectins, monoclonal antibodies and spores of Pasteuria penetrans. Fundam App/ Nemato/ 17, 537-542

Fortuner R, Merny G (1973) Les nématodes parasites des racines associés au riz en basse- Casamance (Sénégal) et en Gambie. Ch ORSTOM, sér Biol 21, 3-20

Hatz B, Dickson DW (1992) Effect of temperature on attachment, development and interactions of Pasteuria penetrans on Meloidogyne arenaria. J Nematol 24, 512-521

Oostendorp M, Dickson DW, Mitchell DJ (1990) Host range and ecology of isolates of Pasteuria spp from the southeastern United States. J Nematol 22, 525531

Oostendorp M, Dickson DW, Mitchell DJ (1991) Population development of Pasteuria penetrans on Meloidogyne arenaria. J Nematol 23, 58-64

Persidis A, Lay JG, Manousis T, Bishop AH, Ellar DJ (1991) Characterization of potential adhesins of the bacterium Pasteuria penetrans, and of putative receptors on the cuticle of Meloidogyne incognita, a nematode host. J Cell Sci 100, 613-622

Prot JC (1979) Horizontal migrations of second stage juveniles of Meloidogyne javanica in sand in concentration gradients of salts and in a moisture gradient. Rev Nematol 2, 17-21

Sayre RM (1980) Biocontrol: Bacillus penetrans and related parasites of nematodes. J Nematol 12, 260270

Sayre RM, Starr MP (1985) Pasteuria penetrans ex Thorne, 1940 nom rev, comb n, sp n, a mycelial and endospore-forming bacterium parasitic in plantparasitic nematodes. Proc Helminth Soc Wash 52 , 149-165

Sayre RM, Wergin WP (1977) Bacterial parasite of a plant nematode: morphology and ultrastructure. J Bacteriol 129, 1091-1101

Seinhorst JW (1950) De betekenis van de toestand von de grond voor het optreden van aanstasting door het stengelaaltje (Ditylenchus dipsaci (Kühn) Filipjev). Tijdschr Plantenziekten 56, 292-349

Seinhorst JW (1962) Modifications of the elutriation method for extracting nematodes from soil. Nematologica 8, 117-128

Stirling GR (1981) Effect of temperature on infection of Meloidogyne javanica by Bacillus penetrans. Nematologica 27, 458-462

Stirling GR (1984) Biological control of Meloidogyne javanica with Bacillus penetrans. Phytopathology 74, 55-60

Stirling GR (1985) Host specificity of Pasteuria penetrans within the genus Meloidogyne. Nematologica 31, 203-209

Stirling GR, Wachtel MF (1980) Mass production of Bacillus penetrans for the biological control of rootknot nematodes. Nematologica 26, 308-312

Stirling GR, Bird AF, Cakurs AB (1986) Attachment of Pasteuria penetrans spores to the cuticles of rootknot nematodes. Rev Nemato/ 9, 251-260

Stirling GR, Sharma RD, Perry J (1990) Attachment of Pasteuria penetrans spores to the root knot nematode Meloidogyne javanica in soil and its effects on infectivity. Nematologica 36, 246-252

Thioulouse $\mathbf{J}$ (1989) Statistical analysis and graphical display of multivariate data on the Macintosh. Computer Applic Biosci 5, 287-292

Verdejo-Lucas S (1992) Seasonal population fluctuations of Meloidogyne spp and the Pasteuria penetrans group in kiwi orchards. Plant Dis 76, 12751279

Wang ELH, Bergeson GB (1974) Biochemical changes in root exudate and xylem sap of tomato plants infected with Meloidogyne incognita. J Nematol 6, 194-202

Zaki MJ, Maqbool MA (1992) Effect of spore concentrations of Pasteuria penetrans on the attachment of Meloidogyne larvae and growth of okra plants. Pakistan J Nematol 10, 69-73 